\title{
Interactive comment on "Historical and future trends in wetting and drying in 291 catchments across China" by Zhongwang Chen et al.
}

\section{Zhongwang Chen et al.}

jjssczw@163.com

Received and published: 18 January 2017

Dear Prof. Michael Roderick,

Thank you for your pertinent comments and kind suggestions on our manuscript entitled "Historical and future trends in wetting and drying in 291 catchments across China" (hess-2015-588). Your previous work (Roderick et al 2014 HESS) does inspire us a lot, and we really treasure your comments on this study. Benefiting from your viewpoint to our study's scientific logic, we have revised it to be more acceptable.

The start point of this study originates from the Peter Greve's study (Greve et al., 2014), in which the DDWW pattern is so attractive that it implies a more uneven distribution of the water availability globally under the climate changes. Though the DDWW pat- 
a fair chance to hold in China. Based on semi centennial observed hydrologic and meteorological data of 291 catchments, we indeed find some results similar to the DDWW pattern. However, we present our findings from an unnatural point to modify the DDWW pattern proposed in Greve's study by adjusting the definition of dry and wet areas based on a threshold in the aridity index. It misleads readers to thinking that the core idea of this study is that the selection of the threshold determines whether the pattern holds or not. In fact, any adjustment to the threshold traps itself in a dilemma where people can always find a different threshold in other regions. Finally, we realize that the uneven trend of the water availability should not be summarized by the DDWW pattern based on a specific threshold, but a statement considering the uncertainty of the threshold. Such statement can be like "drier regions are more likely to become drier, and wetter regions are more likely to become wetter", which may be a universal conclusion around the world, and it is the most significant change in the logic of our study. In our revision, we will present how the revised pattern works in China.

Based on this new logic, we focus on illuminating the fact that the distribution of water resources (runoff) has become more uneven in China since 1950s. In Greve's study, the aridity index is recommended as an indicator of the water availability within a grid for that runoff isn't acquirable in the modelled data. However, since we have the observed streamflow data, the mean annual runoff ( $Q$ İĚ) is a more direct and appropriate choice of reflecting a catchment's water resource condition in this study, which has been neglected in our previous study. The simple framework based on the Budyko hypothesis will still be adopted to model the runoff trend based on the meteorological data in the same period as the observed hydrologic data, revealing the historical runoff change is a response to the change in precipitation basically, as Roderick et al 2014 HESS stated. So the cause of the more uneven trend can be summarized that "more precipitation in wetter areas, and less in drier areas". Furthermore, we concerned about whether the water resources in China will continue to be more uneven in the future, and the simple model provides us an acceptable way to predict future trends based on CMIP5 projected data.

Printer-friendly version

Discussion paper 
We appreciate your advice to re-evaluate our underlying logic of the study. We will add the contents that you suggested to assess the CMIP5 model projections, checking whether precipitation is still the most significant factor in the future. As for the CMIP5 simulations, since we have already acquired the observed data, we think that it is better to use the observed in the process of finding the key factor. And at the same time, the simulations will still be adopted to compare with the observed data in the revised version.

You pointed out that there is non-climate related changes in the runoff in the actual catchments, and asked us for an approach to exact them. In our study, to eliminate the effects of non-climate factors as much as possible, we prudently select the restored streamflow data of catchments that are far away from human activities. Although the effects cannot be removed totally due to the lack of information and technical defects, the restored data are closest to the real natural condition taking all available data into account. We might as well consider it as the real natural runoff (very close), and we can calculate the real natural runoff trend. As for the Budyko-estimated trend, it can be seen as the estimated climate-caused runoff trend, which is the estimated part of the runoff trend directly related to the climate changes. So the residual error between them can be considered as the trend induced by other natural factors, such as land use and vegetation.

Our detailed replies to your comments are listed as follows, and we hope that they are satisfying.

Comment 1: P2, line 10. Why the Greve reference? The original DDWW was Held and Soden 2006?

Comment 2: P 2, line 14, Why the Lim and Greve references? The point about the ocean dominance was originally made by Roderick et al 2014 HESS and was relevant to model projections and not observations.

Printer-friendly version

Comment 3: P. 2, line 18. Why the Roderick reference? That paper did use the phrase 
salt get saltier, etc.., but the underlying results were from a paper by Durack? Perhaps say something like ...... Oceanic observations (Durack et al 2012) confirm a fresh get fresher and salty get saltier pattern (as reinterpreted by Roderick et al 2014 HESS).

Comment 4: P. 2, lines 17-18. Another generalisation relevant here is that rainfall has increased in places with low rainfall and decreased in places with high rainfall (Sun et al 2012 GRL; Donat at al 2016 Nature Climate Change). Comment 7: Eqn 7. Niether Arora 2002 or $\mathrm{Fu}$ et al used that form of the three-term partial differential equation. Why are they cited?

Reply to Comments 1-4 and 7: We are sorry for our carelessness in the paper references and some impertinent summaries of them. In our revision, we will revise the references carefully, and make sure that we can deliver the summaries consistent with the original papers.

Comment 5: P. 4, line 6. You use Penman for PET. The earlier work by Roderick et al 2014 HESS actually followed Budyko and used net irradiance (and not Penman PET). Using Penman PET is not appropriate for vegetated surfaces when $\mathrm{CO} 2$ is changing (e.g. Roderick et al 2015 WRR, Milly and Dunne 2016 Nature Climate Change). For that reason you really need to consider using net radiation. It would be of interest to contrast the net radiation based results with those when the Penman PET is used.

Reply to Comment 5: Thanks for your suggestion, which gives us inspiration for understanding the role of radiation in catchment hydrology, and we will focus on it in the future researches. In this study, considering large regional variation in climatic variables (such as humidity, temperature, and wind speed) in China, we chose Penman equation for estimating PET because it includes effects from humidity and wind speed on PET. We think that Penman equation might have a larger ability in capturing regional variation of atmospheric evaporative demand across China, and the equation has been adopted by previous researches like Yang et al., (2014) and Kai Xu et al., (2015).

Comment 6: Eqn 3. Why c? Later you use n (e.g. Eqn 4). 
Reply to Comment 6: We use c to represent the general parameter that measures the catchment property, while $\mathrm{n}$ can be considered a special $\mathrm{c}$ in Yang's Equation, as in Fu's Equation it becomes " $\omega$ " .

Comment 8: P. 6 line 26. Units. Here and elsewhere. The units of $Q$ are $m m$ a-1. The trend in $Q$ has units $\mathrm{mm}$ a-2. The units of Annual $\mathrm{Q}$ are $\mathrm{mm}$. The key here is that the prefix Annual denotes an integration. The trend in Annual $Q$ has units $\mathrm{mm}$ a-1. So to use those units ( $\mathrm{mm} \mathrm{a-1)}$ for the trend you better put Annual in front of Streamflow at the start of the sentence. Same comment applies throughout.

Reply to Comment 8: It is so nice of you to point out our carelessness in this study again. Actually we hadn't thought over the choice of units until you mentioned in the comment. We indeed confused some concepts and thus their units. We will correct the use of units according to your suggestion

Comment 9: p. 7, line 6. The sentence starting "However, in both situations ...." does not make sense?

Comment 14: P. 11, lines 20-23. This relates to the last comment in the main comments. On page 3, lines 1-2 you correctly point out the need to account for land-use and/or land cover changes. But you did not attempt that. This might be an English problem? Earlier (page 3, line 2) you need to say it is important but here we will ignore it - because that is what you did. Then at the end you need to say - we should not have ignored it (p. 11, lines 20-23). This whole part of the manuscript needs to be explained more clearly.

Reply to Comment 9 and 14: We will carefully modify the manuscript to make any sentences meaningful and our purpose more clear to be caught, trying to avoid English expression problems in our revision.

Comment 10: P. 7, lines 7-11. What is the logic of this? See main comments at the beginning. 
Reply to Comment 10: We will adjust our logic of the study, and the details are shown above.

Comment 11: P. 9, Section 4.2. Why introduce new RESULTS in the DISCUSSION. I did not see the value of this entire section. However, if you want to keep it, then it needs to be moved back to RESULTS.

Comment 12: P. 10, Section 4.3. Same again. You cannot introduce new RESULTS in the DISCUSSION. If you want to keep it, then move it back to the RESULTS.

Reply to Comment 11 and 12: Thanks a lot! Following your comments, we rearranged our sections in the revision by merging Results and Discussion together into a section Results and Discussion to avoid this problem.

Comment 13: Fig. 12. Left Panel. This is truly astonishing. That is the best fit between modelled and observed rainfall I have ever seen! Are you sure of the analysis? I ask because the last sentence of the paper (p. 12, lines 1-2) says that the modelled rainfall was poor? But the results in the left panel of Fig. 12 are truly astonishing. Perhaps I have missed something?

Reply to Comment 13: Thanks for pointing out this issue! After inquiring the data provider from the Institute of Environment and Sustainable Development in Agriculture, the Chinese Academy of Agricultural Sciences, China, we found out the reason why the simulated and observed $P$ fit well, and it should be owned to the bias-correction process. All GCM outputs (precipitation; mean, maximum and minimum air temperature; solar radiation; wind speed; and relative humidity) were bias-corrected according to observations of about 700 meteorological stations around China. The results in Section 3.3 implie the great effectiveness of the correction to $P$ but the failure in outputs related to Ep. Moreover, we realized it is the last sentence of the paper that led to this misapprehension, in which we actually meant to emphasize the uncertainty of GCMs and the deviation between simulated and observed Ep should be concerned instead. Therefore, in our revision, we have deleted the saying that "the modelled rainfall was

Printer-friendly version

Discussion paper 
poor", and focused on the badly simulated Ep.

\section{Reference}

Xu K, Yang D, Yang H, et al. Spatio-temporal variation of drought in China during 19612012: A climatic perspective[J]. Journal of Hydrology, 2015, 526: 253-264. Yang H, Qi $\mathrm{J}, \mathrm{Xu} \mathrm{X}$, et al. The regional variation in climate elasticity and climate contribution to runoff across China[J]. Journal of Hydrology, 2014, 517: 607-616.

comment

Interactive comment on Hydrol. Earth Syst. Sci. Discuss., doi:10.5194/hess-2016-588, 2016. 